\title{
BIM Enclosures, una Aplicación para Cálculos Térmicos en Cerramientos
}

\author{
BIM Enclosures, an Application for Thermal Calculations in Enclosures
}

\author{
Juan Pablo Portillo \\ Universidad de la República, Uruguay \\ jpportillo@farq.edu.uy
}

\begin{abstract}
The BIM software (Building Information Modeling) enables a holistic model -a complete description of the model-building for a complete analysis and validation prior to build, as well as the plotting of final design in architecture and its subsequent maintenance. Users can extend virtually unlimited capabilities. For this reason we have chosen a BIM with programmability by the user to create a calculation module to validate, the heat transfer and possible condensation on both walls and ceilings. The Revit Architecture BIM was chosen using the API (application programming interface) in C\#. In this paper, we analyze the experience gained and the results achieved. The objective is to create a calculation and graphing module thermal transmission and condensation.
\end{abstract}

Keywords: BIM; Cálculo térmico; Condensaciones en muros, Edificios sustentables.

\section{Introducción}

"El fenómeno de Condensación puede producirse en la superficie o en el interior del cerramiento. La presencia de esta patología se manifiesta generalmente por la presencia de hongos, manchas y deterioro o desprendimiento en los materiales de revestimiento" 1

El software BIM (Building Information Modeling o modelado de información Constructiva) posibilita modelar de manera holística una descripción completa del modelo- edificios para un completo análisis y validación previo a construirlo; así como la graficación de proyectos ejecutivos en arquitectura y su posterior mantenimiento. Cuando el software admite la posibilidad adicional de crear módulos de usuario que extienden las capacidades originales del software, se multiplican virtualmente sin límites sus potencialidades. Por esta razón hemos elegido un BIM con capacidad de programación por parte del usuario para crear un módulo de cálculo que permita validar, verificar y graficar la transmisión de calor y posible condensación en cerramientos, tanto muros como techos. EI BIM elegido fue Revit Architecture utilizando el API (Application programming interface) en lenguaje C\#.

En este trabajo pretendemos analizar la experiencia adquirida y los resultados obtenidos. El objetivo del mismo es crear un módulo de cálculo y graficación de transmisiones térmicas y condensaciones en muros y techos aprovechando el modelo BIM el cual permite definir en forma completa el diseño de cerramientos (fig. 1). Mediante lenguaje C\# se crea código que analiza el cerramiento, calcula la transición de calor y las posibles condensaciones graficando los resultados utilizando las

\footnotetext{
${ }^{1}$ de la publicación "Acondicionamiento Térmico", capitulo 3. farq, UdelaR.

herramientas del propio BIM, para lo cual se eligió Autodesk Revit Architecture.

\section{La Importancia del Estudio de Condensaciones}

El estudio de las condensaciones (condensación de agua en muros y techos) y aislación térmica en cerramientos es de vital importancia en el diseño arquitectónico, y más aun si lo enmarcamos en todo lo relativo a sustentabilidad y ahorro energético. este tema que tiene décadas de estudio tiene hoy una vigencia y actualidad fundamental sobre todo considerando los cambios que están ocurriendo a nivel climático, de energía y de desarrollo de proyectos de arquitectura.

Es claro que la condensación acarrea problemas de durabilidad de los materiales, reducción de propiedades de aislación térmica en materiales si estos contienen agua. Incluso existen problemas en la salud de los ocupantes de edificios con este tipo de patologías.

\section{Software Histórico y Necesidad de Actualización}

En los años 90 se produjo un software de cálculo de condensaciones llamado Hterm que realizaba dicho calculo y graficación a partir de una base de datos de materiales. el software fue desarrollado sobre la plataforma Windows. Su desarrollo fue principalmente para uso didáctico a nivel universitario y se descontinuó principalmente debido a que este tipo de proyectos de investigación y desarrollo no cuentan con financiaciones sostenidas a nivel universitario. con el devenir de nuevas versiones del sistema operativo Windows el programa pierde su capacidad de funcionamiento, es decir ya no corre bajo los actuales SO. se plantea así la necesidad de actualizarlo. 
En este escenario y ante la nueva generación de software de modelado BIM surge como opción incorporar este tipo de funciones a Autodesk Revit Architecture.

\section{Cómo Funciona}

La elección de Revit Architecture como plataforma BIM responde a que se trata de un software muy potente que permite realizar una descripción completa del edificio, esto es: modelar la información geométrica tan detallada como se desee así como la información adicional relacionada con los objetos tales como costos, características físicas de los materiales o cantidades de los mismos, posibilita además obtener en tiempo real gráficos de todo el modelo.

Adicionalmente este software permite crear mediante lenguaje C\# entre otros, módulos personalizados que extienden virtualmente al infinito el potencial de cálculo. Esto se logra mediante el API, el cual provee acceso a todos los parámetros de los objetos geométricos del modelo. Este módulo es capaz de leer las características de cada cerramiento elegido en el modelo; de esta manera se aprovecha un modelo que ya existe para un proyecto sin tener que reingresar datos en otra aplicación, luego analiza capa por capa los datos de las mismas y sus materiales para realizar todos los cálculos necesarios.

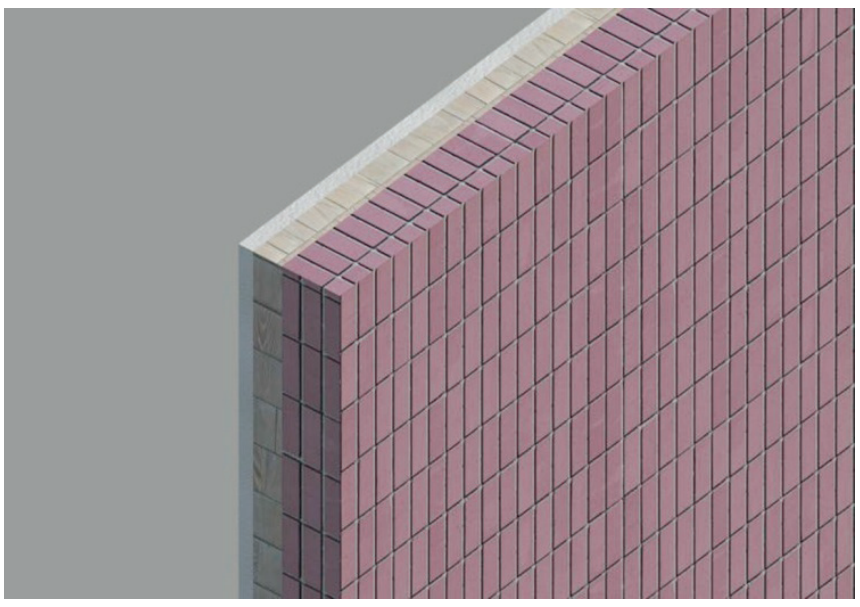

Figura 1: Cerramiento definido en Revit

El módulo desarrollado permite obtener gráficos con el resultado de los cálculos e incorporarlos al conjunto de recaudos que forman parte de la documentación del proyecto tal como se muestra en la imagen (fig. 2). Dicho módulo es de fácil instalación y se accede a él como un nuevo comando en los menús de la interfaz, seleccionando un muro o techo de nuestro proyecto rápidamente se obtienen los resultados de la evaluación de dicho cerramiento (fig. 4). Como ventaja adicional se señala que en la actual versión de Revit Architecture 2013 cada material posee todos los datos físicos necesarios para el cálculo, los mismos se organizan en bloques de información llamados "Assets". Los Assets se conforman de datos tanto mecánicos como térmicos, de esta manera se ha confeccionado una biblioteca de materiales completa de acuerdo a las normas de cálculo para condensaciones en cerramientos.

Una vez que se han analizado todos los datos el modulo procede a realizar los cálculos y genera una vista con los resultados del mismo (fig. 2). los gráficos incluyen la temperatura en cada capa y la temperatura de rocío. El desafío de generar esta nueva aplicación de uso, supone también avanzar en el desarrollo de herramientas que permitan perfeccionar el diseño de edificios sustentables y más eficientes en el uso energético; en este caso para validar cerramientos que posean una capacidad como aislante térmico a la vez que evitar el problema de las condensaciones en capas interiores, patología bastante común cuyas consecuencias son la aparición de hongos en los paramentos interiores entre otras $y$ se deben en general a un diseño defectuoso durante las etapas del proyecto ejecutivo.

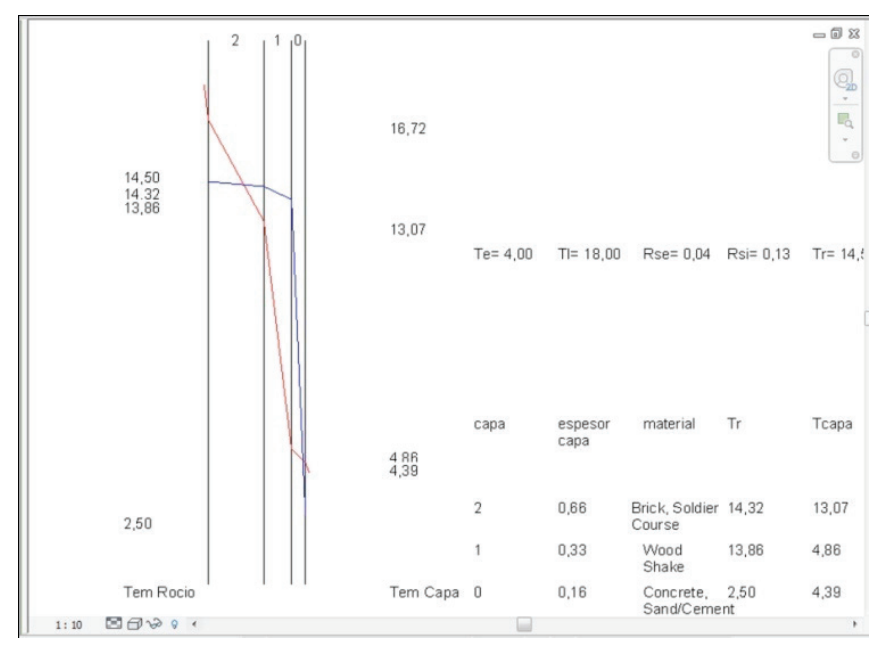

Figura 2: Pantalla de resultados

\section{Código Fuente}

El código se ha desarrollado en lenguaje C\# (fig. 3), el API de Revit en su versión 2013 permite acceder a las características de un objeto del modelo, permitiendo la consulta de por ej. en el caso de un cerramiento de obtener los espesores de cada capa, el material etc.

\section{Estructura del código}

Se define una Clase "CapaCerramiento" que almacena toda la información referida a cada capa del muro o techo, dicha clase se compone de las variables:

public int_identificador; // id de la capa

public double_espesor; // espesor de la capa public string_nombreMat; //nombre del material de la capa 


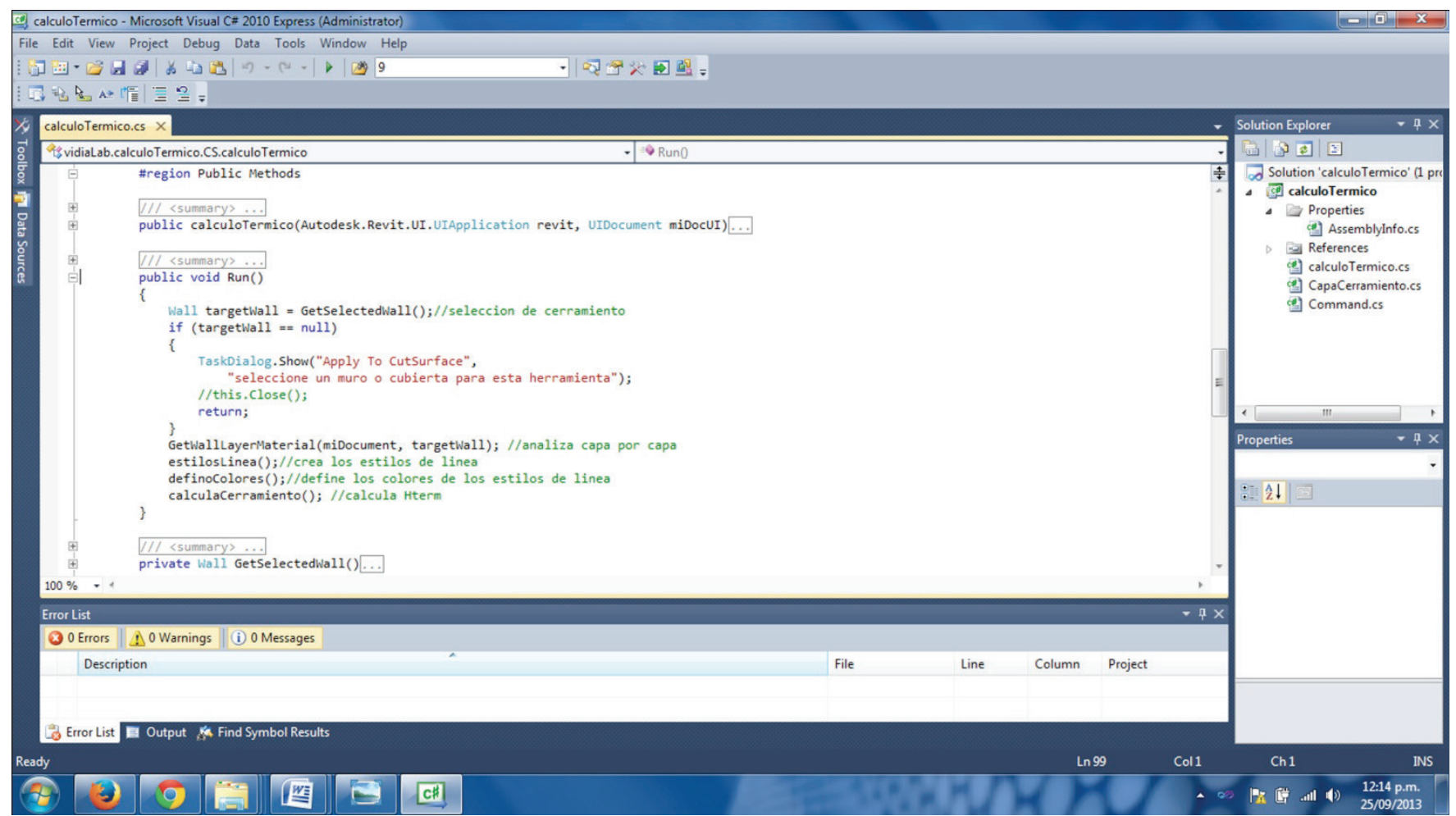

Figura 3: Entorno de desarrollo

public Autodesk.Revit.DB.MaterialFunctionAssignment _funcion;

public double_conductividadTermica;

public double_resistenciaTermica;

public double_permeabilidad;

public double _resistenciaVapor;

public bool_heterogeneo;

Se define la clase que realiza los cálculos y genera las vistas de Revit:

1. Primero se definen todas las variables del proyecto

Autodesk.Revit.DB.Document miDocument; public ArrayList cerramientos = new ArrayList();

Category tipoLinea;

double U; //transmitancia

double $\mathrm{Rt}=0$; //resistencia

double Rsi; //resistencia sup. interior

double RSE $=0.04 ; / /$ resistencia sup. exterior

double $\operatorname{Tr}=14.5 ; / /$ temperatura de rocio

double $\mathrm{TI}=18 ; / /$ temperatura interior

double Te; //temperatura exterior

//double HRI = 80; //humedad relativa interior

//double HRE = 90; //humedad relativa exterior

double Tcapa; // temperatura en la capa

double $\mathrm{PVI}=1652 ; / /$ presión de vapor interior

double Pe; //presion de vapor exterior

double presionVapor = 1652;//presión de vapor

double Rtv;//resistencia total al vapor

int cantidadCapas $=0 ; / /$ cantidad de capas del muro

2. a continuación se definen todas las funciones: public calculoTermico(Autodesk.Revit.UI.UIApplication revit, UIDocument miDocUI)

3. Se define la función principal:

public void Run()//función principal

4. Se definen cada una de las funciones:

private Wall GetSelectedWall()

public void CreateLine(Autodesk.Revit.DB.XYZ startPoint, Autodesk.Revit.DB.XYZ endPoint, ViewDrafting Ventana, string miEstilo)

public void calculaCerramiento()

public void estilosLinea()

public void definoColores()

private double interpolaPresion(double $\mathrm{P}$ )

public void

GetWallLayerMaterial(Autodesk.Revit.DB.Document

document, Wall wall)

Los materiales con los que se construye un modelo de Revit Architecture poseen desde dicha versión datos físicos como por ej. la resistencia térmica.

Mediante código se toman los datos de cada capa y se realizan todos los cálculos de transmisión térmica y condensación para generar un grafico con los resultados obtenidos. 


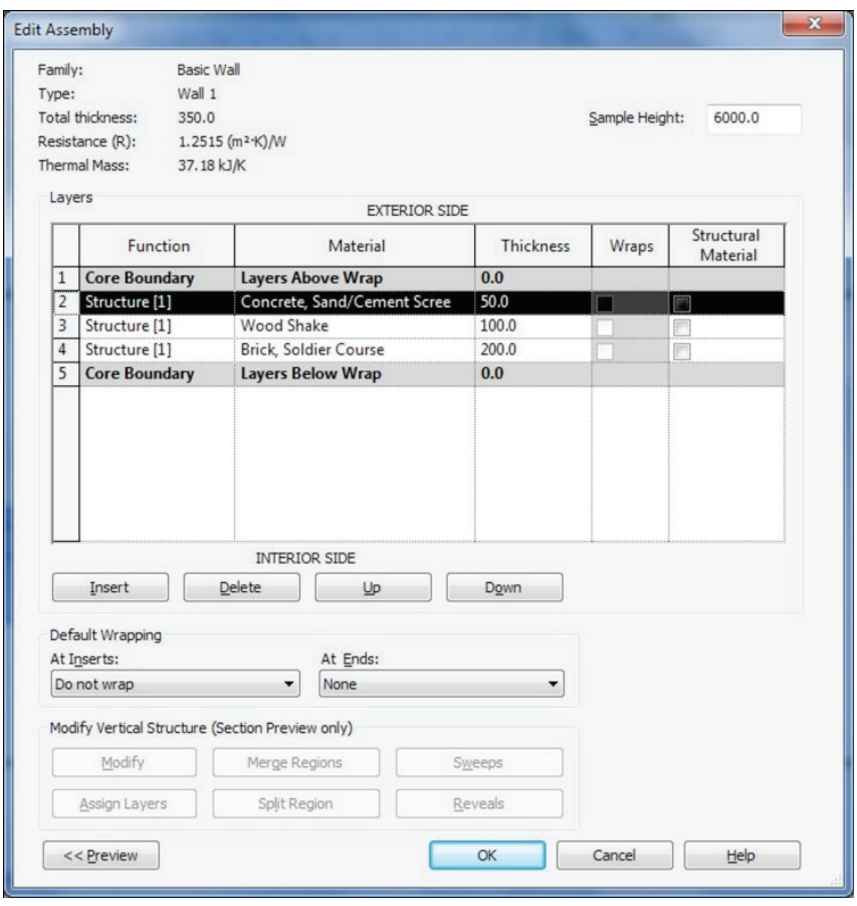

Figura 4: Diseño de muro

Ecuaciones de cálculo:

- Transmitancia: $\mathrm{U}=1 / \mathrm{Rt}, \mathrm{W} /(\mathrm{m} 2 . \mathrm{K})$

- Resistencia total del cerramiento: Rt=Rsi+ $\sum \mathrm{Rca}+\mathrm{Rse}$ $(\mathrm{m} 2 . \mathrm{K}) / \mathrm{W}$

- temperatura en la capa: $\mathrm{t}=\mathrm{t}$ (capa anterior)-Rca.(ti-te)/Rt

- Presión de vapor: P=P2-Rvca.(Pi-Pe)/Rvt

Por una descripción completa de los cálculos ver la citada publicación sobre condensaciones.

\section{Materiales}

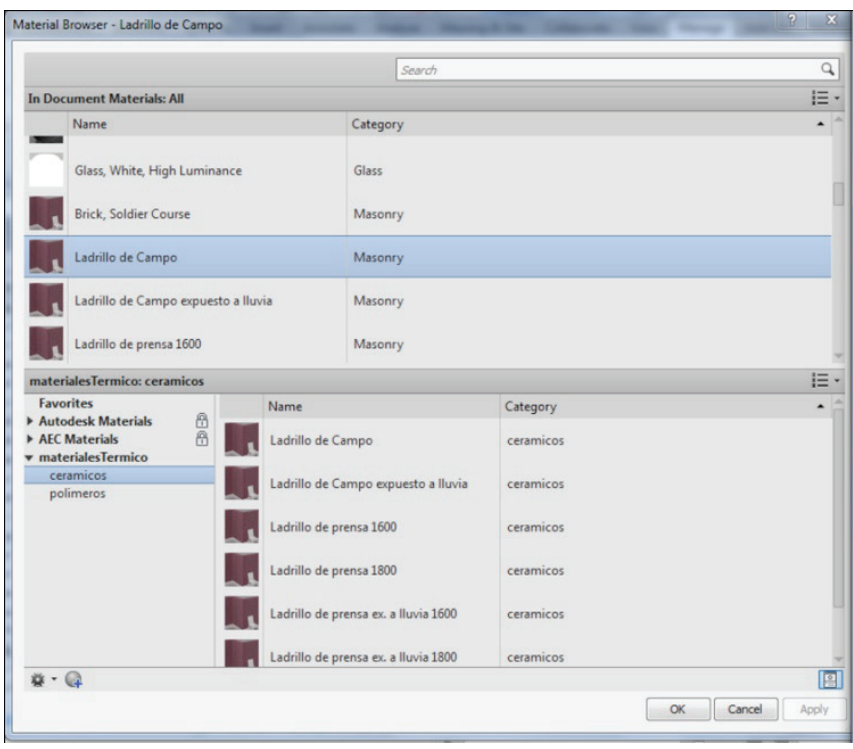

Figura 5: Diseño del material
Los materiales de Revit a partir de la versión 2013 incluyen datos físicos tanto térmicos como mecánicos lo que permite al usuario diseñar y personalizar bibliotecas de materiales (fig. 5 y 6 ). de esta manera se ha confeccionado un catalogo de materiales utilizados en Uruguay que el usuario posee y que puede incluso ampliar. esto permite darle a la aplicación cierta versatilidad.

Como adelantamos más arriba los materiales se organizan en bloques de información llamados "Assets". Los Assets se conforman de datos tanto mecánicos como térmicos, las propiedades térmicas de los materiales son:

- conductividad térmica

- calor específico

- densidad

- emisividad

- permeabilidad

- porsidad

- reflectividad

- Resistividad eléctrica

Se accede desde el código C\# a estos datos para efectuar el cálculo.

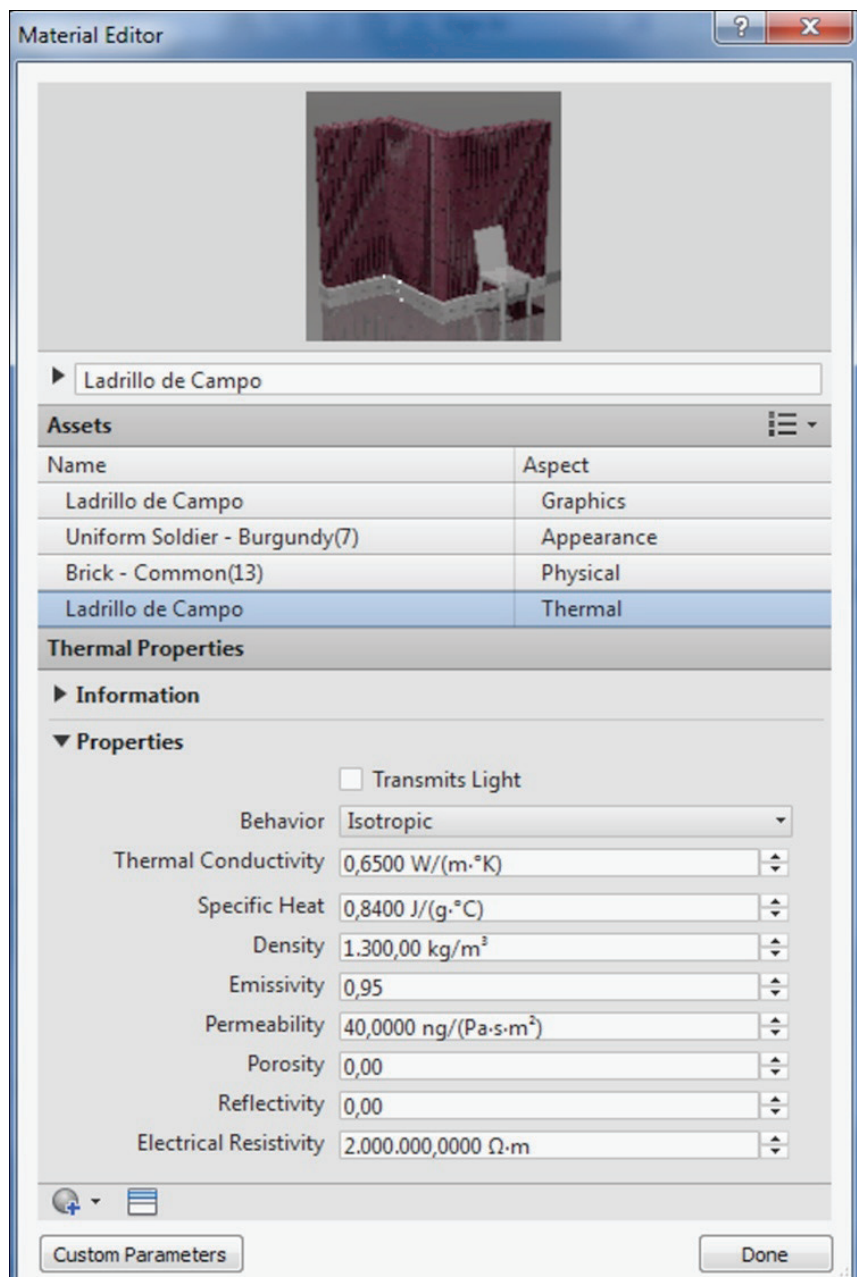

Figura 6: propiedades de los materiales 


\section{Ventajas y Desventajas}

Como ventajas se destaca el hecho de que mediante dicha aplicación es posible evaluar un cerramiento en el contexto de un modelo completo de edificios (BIM) sin la necesidad de utilizar otro software para ello, lo que implica modelar nuevamente los cerramientos. a su vez los resultados y documentación ya forman parte de dicho modelo.

Sin embargo existen al menos por ahora algunos puntos a repensar:

No todas las características de los materiales en términos térmicos están contempladas en el diseño actual de Revit, sobre todo no se tiene en cuenta a los materiales llamados "heterogéneos" como por ej. mampuestos con huecos, tampoco está contemplado el problema de puentes térmicos que se debe encarar como un diseño de otro cerramiento, por ej. cuando existe un elemento puntual en el muro como es el caso de un pilar.

En cuanto a los materiales se suele discriminar en materiales Homogéneos y materiales Heterogéneos desde el punto de vista de del cálculo de la resistencia térmica, en los homogéneos dicha resistencia está relacionada directamente al espesor de la capa, en cambio en los heterogéneos se trabaja habitualmente con un valor equivalente pero no dependiente del espesor ya que la presencia de huecos hace que se comporte diferente.

También se da una situación similar en relación a la permeabilidad al vapor con algunos materiales, por ej. con membranas muy finas cuya permeabilidad no se calcula en función del espesor.

Estas situaciones relacionadas con materiales se tratan como casos particulares y se resuelven de momento a nivel de código.

\section{Trabajo Futuro}

A futuro se puede pensar en integrar la tridimensionalidad en los cálculos, reformular la aplicación teniendo en cuenta las desventajas descritas más arriba e integrar los análisis energéticos de Vasari que se desarrollan cada vez mas, Vasari es una aplicación aun en fase beta de desarrollo que se enmarca dentro de la gama de productos Revit y que permite abordar simulaciones energéticas.

También es clave insertar este proceso en todo lo referente a fabricación digital y la revolución que esta significando la impresión 3D actualmente.

\section{Conclusiones}

Es importante conseguir que el cerramiento mantenga las temperaturas internas lo mas alta posible para evitar riesgos de condensaciones. un correcto diseño es clave para cumplir dicho objetivo. Es responsabilidad del arquitecto que este diseño funcione de manera adecuada.

El uso de herramientas de cálculo directamente vinculadas con un BIM creemos que es estratégico pues permite validar el diseño desde el principio en forma integrada en el software de modelado. los resultados obtenidos con esta herramienta demuestran que es útil y necesaria al momento de elegir como construir un cerramiento que atienda la problemática planteada.

\section{Agradecimientos}

Agradecemos a la cátedra de Acondicionamiento Térmico por toda la información brindada y a la bach. Alejandra Serra por el asesoramiento en el proyecto como estudiante integrante de la cátedra de Acondicionamiento Térmico.

\section{Referencias}

P. Read, J. Vandezande, E. Krygiel. (2013) Masternig Autodesk Revit Architecture. ISBN: 978-1-1182-4478-4 2013 Wiley.

Autodesk, Inc. (2012) Revit 2012 API Developer's Guide.

A. Picción, M. Camacho, G. Cheirasco, R. Tanca, M. N. Lopez. (2004). Guión 2, Publicación de la cátedra de A. térmico de la Facultad de Arquitectura de la UdelaR.

Rivero, Aroztegui, Girardin, Musso, (2002). Repartido AT01, cátedra de A. térmico, farq. 